\title{
Representación Simultánea del Equilibrio Líquido-Vapor, el Volumen Molar y la Entalpía de Exceso de Mezclas Complejas mediante una Ecuación de Estado tipo Peng-Robinson
}

\author{
Luis A. Forero* y Jorge A. Velásquez \\ Facultad de Ingeniería Química. Universidad Pontificia Bolivariana. AA 56000. Medellín-Colombia \\ (e-mail: luis.foreroga@upb.edu.co; jorge.velasquezj@upb.edu.co) \\ * Autor a quien debe ser dirigida la correspondencia.
}

Recibido Mar. 27, 2019; Aceptado May. 24, 2019; Versión final Jun. 28, 2019, Publicado Dic. 2019

\begin{abstract}
Resumen
Una ecuación de estado generalizada tipo Peng-Robinson con las reglas de mezclado de Huron-Vidal se emplea para correlacionar simultáneamente el equilibrio liquido-vapor, el volumen molar y las entalpías de exceso de mezclas polares y no polares. Se usa el concepto de series homólogas para desarrollar expresiones generalizadas que permitan representar las propiedades termodinámicas de una mezcla a partir de parámetros de las sustancias puras que la conforman. Se analizan las siguientes series homólogas: etanol+n-alcanos, butanol+n-alcanos, 2-metoxi-2-metilbutano+n-alcanos, dimetil carbonato+n-alcanos, 2metoxi-2-metilbutano+n-alcoholes y dimetil carbonato $+n$-alcoholes. Expresiones generalizadas en términos de la razón entre la temperatura crítica y la presión crítica de los componentes de la serie homologa, fueron desarrolladas para obtener los parámetros de interacción binaria de una mezcla. En general, los resultados son aceptables e ilustran la capacidad del modelo termodinámico empleado para representar las tres propiedades de forma simultánea.
\end{abstract}

\section{Simultaneous Representation of Liquid-Vapor Equilibria, Molar Volume and Excess Enthalpy of Complex Mixtures by a Peng- Robinson-type Equation of State}

\begin{abstract}
A generalized Peng -Robinson equation of state with the Huron-Vidal mixing rules is selected to correlate vapor-liquid equilibrium, molar volume and excess enthalpy of polar and non-polar mixtures. The concept of homologous series is used to develop generalized expressions that can be used to describe the thermodynamic properties of a binary mixture from parameters of the pure substances. The homologous series analyzed are: ethanol+n-alkanes, butanol+n-alkanes, 2-methoxy-2-methylbutane+n-alkanes, dimethyl carbonate $+n$-alkanes, 2-methoxy-2-methylbutane $+n$-alcohols and dimethyl carbonate $+n$-alcohols. Generalized expressions to obtain the binary interaction parameters of a mixture were developed as a function of the ratio between the critical temperature and the critical pressure of the compounds in the homologous series. In general, results are acceptable and show the ability of the thermodynamic model to represent the three properties simultaneously.
\end{abstract}

Keywords: Peng -Robinson; Huron-Vidal; cubic EoS; excess enthalpy; VLE; molar volume 


\section{INTRODUCCIÓN}

En el año 2017 el consumo de energía mundial alcanzó los 157.000 GWh/año con un crecimiento del 2.2\% anual (British Petroleum Company, 2018). Los hidrocarburos convencionales se están agotando, serán muy costosos en el futuro y su potencial contaminante es cada vez más inaceptable. Para superar estos problemas se requieren nuevos combustibles o aditivos (Beatrice et al., 2013; Fantozzi et al., 2016). Los combustibles oxigenados usados como aditivos muestran un gran potencial para reducir contaminantes en las máquinas de combustión interna. Entre los aditivos oxigenados más comunes están el etanol, el butanol, el dimetil carbonato (DMC) y el 2-metoxi-2-metilbutano (TAME). Por lo tanto, una predicción precisa del equilibrio de fases, entalpías de mezcla y volúmenes molares en mezclas de estos aditivos con hidrocarburos y alcoholes es de gran interés para la industria del petróleo.

De acuerdo con Kontogeorgis y Coutsikos (2012) existen pocos estudios acerca del uso de las reglas de mezclado del tipo EdE $+\mathrm{GE}^{\mathrm{E}}$ para describir propiedades como la entalpía de exceso en comparación con los estudios realizados en el tema de equilibrio de fases. La principal aplicación de las ecuaciones cúbicas de estado corresponde a la descripción del equilibrio de fases (Cismondi y Mollerup, 2005). Sin embargo, algunos autores han utilizado este tipo de modelos termodinámicos para describir de forma simultánea el equilibrio y la entalpía de exceso. En la Tabla 1 se presenta un resumen de los pocos trabajos que se han realizado en este campo para las reglas de mezclado del tipo $\mathrm{EdE}+\mathrm{GE}$.

Tabla 1. Principales trabajos acerca del ajuste simultáneo del equilibrio de fases y la entalpía de exceso con reglas del tipo EdE+GE.

\begin{tabular}{|c|c|c|c|}
\hline $\mathrm{N}^{\circ}$ & Naturaleza de las mezclas & Modelos utilizados & Referencia \\
\hline 11 & $\begin{array}{l}\text { agua-polar } \\
\text { Polar-Polar } \\
\text { Polar- benceno } \\
\text { Acetonitrilo-alcano }\end{array}$ & $\begin{array}{l}\text { PRSV-MHV1-NRTL } \\
\text { PRSV-WS-NRTL }\end{array}$ & Ohta, 1997 \\
\hline 4 & $\begin{array}{l}\text { isopropanol-agua } \\
\text { metanol-benceno } \\
\text { benceno-ciclohexano } \\
\text { acetona-agua }\end{array}$ & $\begin{array}{l}\text { PRSV con } \\
\text { WS, HV, LCVM, MHV2 }\end{array}$ & Orbey y Sandler, 1996 \\
\hline 8 & $\begin{array}{l}\text { dióxido de carbono-alcohol } \\
\text { metanol-alcano } \\
\text { tetrahidrofurano-DMF } \\
\text { tetrahidrofutano-ciclohexano } \\
\text { DMF-ciclohexano } \\
\text { benceno-ciclohexano } \\
\text { ciclopentano-tetracloroetileno }\end{array}$ & PRSV-WS-NRTL & $\begin{array}{l}\text { Escobedo-Alvarado y } \\
\text { Sandler, } 2001\end{array}$ \\
\hline 6 & $\begin{array}{l}\text { etanol-ciclohexano } \\
\text { etanol-tetrahidrofurano } \\
\text { tetrahidrofurano-ciclohexano } \\
\text { DMF-ciclohexano } \\
\text { DMF-tetrahidrofurano } \\
\text { cloroformo-tetrahidrofurano }\end{array}$ & PRSV-HV-NRTL & Djorjevic et al.,1999 \\
\hline 9 & alcohol-alcano & PRSV-WS-Shen y Lu & Shen y Lu, 1997 \\
\hline 10 & $\begin{array}{l}\text { alcanos-aromáticos } \\
\text { alcanos-cetonas } \\
\text { aromáticos-cetonas }\end{array}$ & VTPR-GC & Ahlers y Gmehling, 2002 \\
\hline 4 & ester-alcano & VTPR-GC & Collinet y Gmehling, 2005 \\
\hline 7 & $\begin{array}{l}\text { cetona-alcano } \\
\text { alcohol-alcano } \\
\text { aromático-alcano }\end{array}$ & UMR-GC & Voutsas et al., 2006 \\
\hline 4 & dieter-alcano & $\begin{array}{l}\text { PRSV-MHV1-NRTL } \\
\text { PRSV-MHV2-NRTL }\end{array}$ & Šerbanović et al., 2004 \\
\hline
\end{tabular}

De acuerdo con la Tabla 1 el ajuste simultáneo ha sido estudiado para una cantidad muy pequeña de mezclas. Ninguno de los autores ha analizado más de 11 mezclas y en total existen resultados reportados para 63 mezclas. Vale la pena destacar que en la mayoría de los casos se ha estudiado la correlación de ambas propiedades mediante el ajuste de cada mezcla estudiada. Solo en los trabajos de Gmehling (Ahlers y Gmehling, 2002; Collinet y Gmehling, 2005) y de Voutsas et al. (2006), se ha incluido el desarrollo de modelos 
generalizados mediante el concepto de contribución de grupos. En cuanto a la naturaleza de las mezclas, no se han cubierto una cantidad de grupos funcionales considerables. En el campo de interés de las gasolinas oxigenadas destacan los resultados reportados por Shen y Lu (1997) para alcoholes con alcanos. Sin embargo, no hay reporte de trabajos para mezclas del tipo aditivo oxigenado-alcano o aditivo oxigenadoalcohol.

Por otro lado, la conclusión general es que se debe trabajar con parámetros dependientes de la temperatura si se desea correlacionar ambas propiedades. De acuerdo con Orbey y Sanlder (1996) no es posible predecir correctamente la entalpía de exceso a partir del ajuste de equilibrio. El caso contrario tampoco ofrece buenos resultados. La única manera de obtener buenos resultados es mediante la minimización de una función objetivo que tenga en cuenta la desviación del equilibrio y la desviación en la entalpía de exceso (Ohta,1997; Orbey y Sandler, 1996).

El propósito principal de este artículo consiste en evaluar la capacidad de una ecuación de estado cúbica del tipo Peng-Robinson con reglas de mezclado Huron-Vidal-NRTL para representar simultáneamente el equilibrio de fases, las entalpías de exceso y los volúmenes de mezclas que contienen sustancias polares y no polares. Como se muestra en publicaciones recientes (Forero y Velásquez, 2016b; Velásquez y Forero, 2017) el modelo termodinámico seleccionado describe correctamente el equilibrio líquido-vapor y líquidolíquido de mezclas de alcanos con compuestos polares. Se trabajará con mezclas del tipo aditivo oxigenadoalcano o aditivo oxigenado-alcohol lo cual constituye una novedad y los resultados obtenidos serán de interés para la industria petrolera. Se emplea el concepto de series homólogas para desarrollar expresiones generalizadas que permitan la predicción de diferentes propiedades termodinámicas a partir de parámetros de las sustancias puras que conforman una mezcla.

\section{MODELO TERMODINÁMICO}

La ecuación de estado es del tipo Peng-Robinson trasladada en el volumen que puede plantearse como sigue (Forero y Velásquez, 2016a):

$$
P=\frac{R T}{v+c-b}-\frac{a}{v^{2}+2 v(c+b)+c^{2}+2 c b-b^{2}}
$$

Los parámetros $\mathrm{a}, \mathrm{b}$ y $\mathrm{c}$ de la mezcla se pueden calcular a partir de las reglas de mezcla de Huron-Vidal. La regla de mezcla utilizada combina las fortalezas de los modelos de actividad (modelamiento de equilibrio de fases de mezclas complejas a bajas presiones) con las fortalezas de las EdEs cúbicas (modelamiento de equilibrio de fases de mezclas simples a altas presiones) (Kontogeorgis y Coutsikos, 2012). El punto de arranque para derivar la mayoría de estas reglas de mezcla viene de igualar la $\mathrm{G}^{\mathrm{E}}$ que se obtiene a partir de la EdE cúbica con la GE del modelo de actividad a una presión dada. Las reglas de mezcla de Huron-Vidal fueron las primeras de este tipo (Huron y Vidal, 1979), la cual utiliza como referencia una presión infinita. La selección de estas reglas de mezcla sobre otras disponibles se discute ampliamente en otra publicación (Forero y Velásquez, 2016b). Utilizando el modelo de actividad NRTL el cálculo de los parámetros a, b y c de la mezcla con estas reglas es como sigue:

$$
\begin{aligned}
& a=b\left[\sum_{i}^{N C} x_{i} \frac{a_{i}}{b_{i}}-\frac{G^{E}}{0.6232}\right] \\
& b=\sum_{i}^{N C} x_{i} b_{i} \\
& c=\sum_{i}^{N C} x_{i} C_{i} \\
& G^{E}=R T \sum_{i}^{N C} x_{i}\left(\frac{\sum_{j}^{N C} x_{j} \frac{A_{j i}}{R T} \exp \left(-\alpha_{j i} \frac{A_{j i}}{R T}\right)}{\sum_{k}^{N C} x_{k} \exp \left(-\alpha_{k i} \frac{A_{k i}}{R T}\right)}\right)
\end{aligned}
$$


Las ecuaciones (2), (3) y (4) constituyen las reglas de mezcla. La ecuación (5) representa la expresión para $G^{E}$ según el modelo NRTL (Renon y Prausnitz, 1968). Los parámetros de las sustancias puras $a_{i}, b_{i}$ y $c_{i}$ se calculan a partir de la temperatura del sistema, las propiedades críticas $\left(\mathrm{T}_{\mathrm{Ci}}, \mathrm{P}_{\mathrm{Ci}}\right)$, el factor acéntrico $\omega_{\mathrm{i}} \mathrm{y}$ el factor polar de Halm-Stiel (1967) $\chi_{i}$ mediante las siguientes expresiones:

$$
\begin{aligned}
& \omega_{i}=-1-\left.\log \frac{P^{s a t}}{P_{C}}\right|_{T_{r}=0.7} \\
& \chi_{i}=\left.\log \frac{P^{s a t}}{P_{c}}\right|_{T_{r}=0.6}+1.7 \omega_{i}+1.552 \\
& a_{i}=0.45723553 \frac{\left(R T_{C i}\right)^{2}}{P_{C i}} \exp \left[H_{1 i}\left(1-\left(\frac{T}{T_{C i}}\right)^{H_{2 i}}\right)\right] \\
& b_{i}=0.07779607 \frac{R T_{C i}}{P_{C i}} \\
& c_{i}=\left[v_{\text {Notransladada }}^{L}-v_{\text {Experimental }}^{L}\right]_{T_{r}=0.7} \\
& H_{2 i}=\frac{\gamma_{c i}}{\beta_{c i}-1}+\beta_{c i} \\
& H_{1 i}=\frac{\beta_{c i}-1}{H_{2 i}} \\
& \beta_{c i}=1.34695+1.49228 \omega_{i}+1.4252 \chi_{i}-0.12039 \omega_{i}^{2}+2.8166 \chi_{i}^{2}+0.312707 \omega_{i} \chi_{i} \\
& \gamma_{c i}=-0.37592-1.44144 \omega_{i}+6.20445 \chi_{i}-0.67473 \omega_{i}^{2}+15.586 \chi_{i}^{2}-0.70582 \omega_{i} \chi_{i}
\end{aligned}
$$

Las ecuaciones (6) y (7) son las definiciones del factor acéntrico y el factor polar respectivamente. La ecuación (8) muestra el cálculo de ai utilizando la función alfa propuesta por Heyen (1980). La ecuación (9) ilustra el cálculo de $b_{i}$. El parámetro de traslación $c_{i}$ se calcula según la ecuación (10) requiriendo un volumen molar experimental para el líquido a $\mathrm{T}_{\mathrm{r}}=0.7$. Las ecuaciones (11) a (14) muestran como calcular los parámetros de la función alfa de Heyen en términos de los factores acéntrico y polar. Los parámetros $\beta_{c i}$ y $\gamma_{c i}$ están relacionados con la primera y segunda derivada de la función alfa evaluada a la temperatura crítica (Forero y Velásquez, 2016a).

\section{MEZCLAS DE INTERÉS EN EL CAMPO DE GASOLINAS OXIGENADAS}

Con el objetivo de expresar los coeficientes de interacción binaria de las reglas de mezclado Huron-VidalNRTL como una función de la temperatura, se utilizó la siguiente expresión cuadrática para los parámetros de energía de interacción del modelo NRTL:

$$
A_{j i}=a_{j i}+b_{j i} T+c_{j i} T^{2}
$$

En la ecuación (15), A A ri representa los parámetros de interacción de la ecuación (5). Los coeficientes aji, bji y $\mathrm{C}_{\mathrm{ji}}$ dependen de cada mezcla. La ecuación (15) es la sugerida y empleada por Ahlers y Gmehling (2002) en el método de contribución de grupos VTPR. Con respecto a las mezclas estudiadas, se trabajó con las siguientes series homólogas: etanol-alcanos, butanol-alcanos, TAME-alcanos, DMC-alcanos, TAME-alcoholes y DMCalcoholes. En total se analizaron 26 mezclas binarias. De la misma forma que para el equilibrio líquido-vapor (Forero y Velasquez, 2016b), se eligió el factor $R T_{c} / P_{c}$ de la serie homóloga de los alcanos o de los alcoholes para obtener expresiones generalizadas para $a_{j i}, b_{j i}$ y $c_{j i}$, de tal forma que la expresión general para cada parámetro es de la forma: 


$$
p_{j i}=m_{j i}\left(\frac{R T_{c}}{P_{c}}\right)_{S . H .}^{2}+n_{j i}\left(\frac{R T_{c}}{P_{c}}\right)_{S . H .}+o_{j i}
$$

En la ecuación (16) $\mathrm{p}_{\mathrm{ji}}$ representa cualquiera de los coeficientes $\mathrm{a}_{\mathrm{ji}}$, $\mathrm{b}_{\mathrm{ji}}$ ó $\mathrm{c}_{\mathrm{ji}}$ de la ecuación (15). El factor $\mathrm{RT} / \mathrm{P}_{\mathrm{c}}$ es el de cada miembro de la serie homóloga. Este factor es proporcional al covolumen bi definido en la ecuación 9 y que permite diferenciar el tamaño de las moléculas entre los miembros de una serie homóloga. En principio, para cada serie homóloga, se deben estimar 18 parámetros. Sin embargo, para cada serie inicialmente se estudió la posibilidad de trabajar con expresiones generalizadas lineales. En este caso el ajuste de datos implica la estimación de 12 parámetros. Para el parámetro de mezclado aleatorio $\left(\alpha_{\mathrm{j}}\right)$ se emplearon dos tipos de expresiones. Para las mezclas con la serie de alcanos, se emplea la misma expresión desarrollada para describir el equilibrio líquido-vapor de mezclas de alcoholes con alcanos (Forero y Velásquez, 2016b). La expresión utilizada es:

$$
\alpha_{12}=-0.065\left(\frac{R T_{c}}{P_{c}}\right)_{\text {alcanos }}+0.459
$$

Para mezclas con la serie homóloga de alcoholes por simplicidad, el valor de $\alpha_{\mathrm{ji}}$ se toma constante e igual a 0.3 , ya que este parámetro tiene poca influencia en los cálculos ELV en un amplio rango de 0.2 a 0.47 (Zhao et al., 2017). Por ejemplo, para el ELV de la mezcla etanol-DMC a $40 \mathrm{kPa}$, variar el alfa de 0.2 a 0.47 cambia la DAP de $0.15 \%$ a $0.16 \%$ y para el ELV de la mezcla etanol-TAME a $323.15 \mathrm{~K}$ y $333.15 \mathrm{~K}$, variar el alfa de 0.2 a 0.47 cambia la DAP de $0.40 \%$ a $0.21 \%$. Es importante aclarar que las DAP mencionadas corresponden solo al ajuste de datos de ELV. Los parámetros de cada serie homóloga corresponden a aquellos que minimizan la siguiente función objetivo:

$$
\text { F.O. }=0.8 \sum_{j=1}^{N S} \sum_{i=1}^{N D_{j}}\left|\frac{P_{b \exp }^{i}-P_{b E d E}^{i}}{P_{b \exp }^{i}}\right|_{j}+0.2 \sum_{j=1}^{N S} \sum_{i=1}^{N D_{j}}\left|\frac{H_{\mathrm{exp}}^{E i}-H_{E d E}^{E i}}{H_{\exp }^{E i}}\right|_{j}
$$

La ecuación (18) se encuentra formada por el error total relativo en la presión de burbuja y la entalpía de exceso. Los factores de peso se fijan de manera arbitraria. En este caso se ha elegido un valor de 0.8 para el error en la presión de burbuja con el objetivo de darle prioridad al equilibrio de fases sobre la entalpía de exceso. La función objetivo que se ha empleado es similar a la utilizada por Ohta et al. (1997). La minimización de la ecuación (18) se realizó para las 6 series estudiadas. Las expresiones generalizadas desarrolladas se reportan en la Tabla 2. Para las tres series con $n$-alcanos se encontró que es suficiente con simplificar la ecuación (16) a una expresión lineal. Para conseguir el ajuste simultáneo de la entalpía y la presión de burbuja de la serie DMC con alcoholes, fue necesario emplear la expresión cuadrática.

Los resultados para el ELV y la entalpía de exceso luego del ajuste de cada una de las series analizadas se presentan en la Tabla 3. En la tabla también se incluyen los resultados para la predicción del volumen molar. Los datos experimentales utilizados están disponibles en la literatura, sin embargo, dada la gran cantidad de referencias estas se han omitido. Para el lector interesado las mismas pueden ser solicitadas a los autores. Para cada mezcla se calcula la desviación absoluta promedio (DAP) para la propiedad de interés (Y) tal como se define en la ecuación (19):

$$
D A P=\frac{1}{N D} \sum_{i=1}^{N D}\left|\frac{Y_{b \exp }^{i}-Y_{b E d E}^{i}}{Y_{b \exp }^{i}}\right| 100
$$

En resumen, los resultados ilustran la capacidad de la nueva EdE para representar las tres propiedades de forma simultánea. Las desviaciones globales (promedio de la DAP para todas las mezclas) son $0.99 \%, 3.85 \%$ y $0.92 \%$ para la presión de burbuja, la entalpía de exceso y el volumen molar respectivamente. El análisis de las DAPs de cada propiedad tiene las siguientes características: Para el ELV, la DAP en la presión de burbuja se encuentra entre $0.10 \%$ y $2.68 \%$. El $69 \%$ de las mezclas analizadas presenta una DAP por debajo de $1.0 \%$. Por otra parte, la desviación absoluta en la fracción molar del vapor tiene un valor medio de 0.007 . Para la entalpía de exceso la DAP se encuentra entre $1.42 \%$ y $6.30 \%$. En el $54 \%$ de los casos la DAP tiene un valor inferior a $3.85 \%$. La DAP en el volumen se encuentra entre $0.15 \%$ y $1.96 \%$. La DAP es menor a $1.00 \%$ para el $65 \%$ de las mezclas. La mayor desviación global para la entalpia de exceso es normal y se puede atribuir a las debilidades inherentes de las ecuaciones de estado cúbicas para predecir entalpías en fase líquida (Valderrama, 2003). 
Tabla 2. Parámetros generalizados de la nueva EdE para las series homólogas.

$$
x=\left(\frac{R T_{c}}{P_{c}}\right)_{\text {alcololes }}, y=\left(\frac{R T_{c}}{P_{c}}\right)_{\text {alcanos }}
$$

\begin{tabular}{|c|c|c|c|c|}
\hline 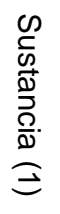 & 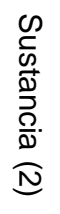 & $\mathrm{a}_{\mathrm{ji}}(\mathrm{kJ} / \mathrm{kmol})$ & $b_{j i}(\mathrm{~kJ} / \mathrm{kmol} \mathrm{K})$ & $\left.\mathrm{C}_{\mathrm{ji}}(\mathrm{kJ} / \mathrm{kmol} \mathrm{K})^{2}\right)$ \\
\hline 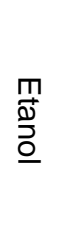 & 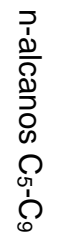 & $\begin{array}{l}a_{12}=1032 y-2820 \\
a_{21}=-234 y-14530\end{array}$ & $\begin{array}{l}b_{12}=10.5 y+14.7 \\
b_{21}=6.62 y+127.62\end{array}$ & $\begin{array}{l}c_{12}=0.0015 y-0.0292 \\
c_{21}=-0.0304 y-0.1642\end{array}$ \\
\hline 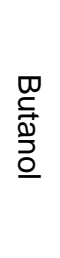 & 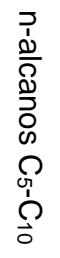 & $\begin{array}{l}a_{12}=744 y-1487 \\
a_{21}=202 y-20906\end{array}$ & $\begin{array}{l}b_{12}=4.0 y+3.6 \\
b_{21}=2.4 y+166.50\end{array}$ & $\begin{array}{l}c_{12}=0.0014 y-0.0076 \\
c_{21}=-0.0126 y-0.2538\end{array}$ \\
\hline $\begin{array}{l}-1 \\
\frac{3}{3} \\
\text { m }\end{array}$ & 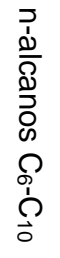 & $\begin{array}{l}a_{12}=-30,3 y+986 \\
a_{21}=-1098 y+3696\end{array}$ & $\begin{array}{l}b_{12}=7.0 y-17.6 \\
b_{21}=2.8 y-11.3\end{array}$ & $\begin{array}{l}c_{12}=-0.0181 y+0.0459 \\
c_{21}=-0.0007 y+0.0031\end{array}$ \\
\hline ᄁo & 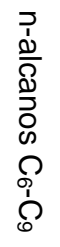 & $\begin{array}{l}a_{12}=3928 y-9.5 \\
a_{21}=2828 y+9674\end{array}$ & $\begin{array}{l}b_{12}=-0.123 \\
b_{21}=-9.52 y-39\end{array}$ & $\begin{array}{l}c_{12}=-0.0031 y-0.0079 \\
c_{21}=0.0065 y+0.0478\end{array}$ \\
\hline 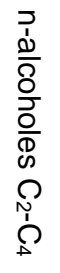 & $\underset{\substack{n \\
\frac{1}{3}}}{-1}$ & $\begin{array}{l}a_{12}=-1098 x+920 \\
a_{21}=140 x+3450\end{array}$ & $\begin{array}{l}b_{12}=-0.27 x-0.005 \\
b_{21}=0.0025\end{array}$ & $\begin{array}{l}c_{12}=-0.0349 x+0.0363 \\
c_{21}=0.0007 x-0.0011\end{array}$ \\
\hline 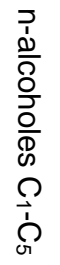 & ᄁุ & $\begin{array}{l}a_{12}=-9190 x^{2}+18270 x-6018 \\
a_{21}=-1615 x^{2}+5431 x+2694\end{array}$ & $\begin{array}{l}b_{12}=1.4 x^{2}-1.7 x+0.5 \\
b_{21}=-0.48 x+0.74\end{array}$ & $\begin{array}{l}c_{12}=0.226 x^{2}-0.422 x+0.189 \\
c_{21}=-0.067 x^{2}+0.089 x-0.063\end{array}$ \\
\hline
\end{tabular}

En la Figura 1 se presenta la DAP de cada propiedad para cada una de las seis series estudiadas. De acuerdo con la figura solo para la serie alcohol-TAME se obtiene una DAP cercana al $2.00 \%$ en la presión de burbuja. Para la entalpía de exceso tres de las seis series tienen una DAP entre el $4.0 \%$ y 5.0\%. La mayor desviación corresponde a la serie etanol-alcanos. Finalmente, los resultados del volumen son muy buenos para todas las series. En los seis casos las DAPs son cercanas o se encuentran por debajo de 1.0\%. 
Tabla 3. Resultados para el equilibrio líquido-vapor, la entalpía de exceso y el volumen de mezclas con la nueva EdE.

\begin{tabular}{|c|c|c|c|c|c|c|c|c|c|c|c|c|}
\hline \multirow[b]{2}{*}{ Sustancia 1} & \multirow[b]{2}{*}{ Sustancia 2} & \multicolumn{5}{|c|}{ ELV } & \multicolumn{3}{|c|}{$\mathrm{H}^{\mathrm{E}}$} & \multicolumn{3}{|c|}{ v } \\
\hline & & ND & $\mathrm{T}(\mathrm{K})$ & $\mathrm{P}(\mathrm{kPa})$ & DAP P & Dy & ND & $\mathrm{T}(\mathrm{K})$ & $\mathrm{DAP} \mathrm{H}$ & ND & $\mathrm{T}(\mathrm{K})$ & DAP v \\
\hline \multirow{5}{*}{ etanol } & pentano & 26 & 303 & $10.5-87$ & 0.70 & 0.003 & 24 & 298 & 6.16 & 9 & 298 & 0.64 \\
\hline & hexano & 50 & $298-333$ & $18-107$ & 0.81 & 0.006 & 125 & $283-318$ & 4.43 & 9 & 298 & 0.81 \\
\hline & heptano & 92 & $303-343$ & $7.8-96$ & 0.77 & 0.006 & 40 & $298-318$ & 4.49 & 9 & 298 & 1.11 \\
\hline & octano & 56 & $313-348$ & $4.1-95$ & 0.89 & 0.004 & 38 & $298-318$ & 5.06 & 9 & 298 & 1.65 \\
\hline & nonano & 27 & 343 & $6.3-73$ & 0.61 & 0.001 & 39 & $298-318$ & 5.68 & & & \\
\hline \multirow{5}{*}{ butanol } & pentano & 15 & 303 & $1.3-82$ & 1.48 & 0.003 & 15 & 298 & 3.52 & 11 & 298 & 0.43 \\
\hline & hexano & 56 & $288-333$ & $0.4-77$ & 1.48 & 0.003 & 28 & $288-298$ & 6.22 & 42 & $298-308$ & 0.51 \\
\hline & heptano & 69 & $313-363$ & $2.5-89$ & 0.98 & 0.005 & 31 & $288-328$ & 5.74 & 76 & $288-318$ & 0.87 \\
\hline & octano & 40 & $308-373$ & $2.4-73$ & 1.23 & 0.016 & 34 & $288-328$ & 6.30 & 42 & $298-308$ & 1.34 \\
\hline & decano & 42 & $358-388$ & $5.1-93$ & 0.77 & 0.008 & 56 & $288-298$ & 2.74 & 42 & $298-308$ & 1.93 \\
\hline \multirow{4}{*}{ TAME } & hexano & 26 & 313 & 19.6-37 & 0.34 & 0.001 & 49 & $298-313$ & 1.62 & 18 & 298 & 0.83 \\
\hline & heptano & 26 & 313 & 12.3-19.6 & 0.10 & 0.001 & 18 & $298-313$ & 4.97 & & & \\
\hline & octano & 18 & 323 & $6.7-29.3$ & 0.61 & 0.003 & 18 & $298-313$ & 3.39 & 19 & 298 & 1.36 \\
\hline & decano & 77 & $308-328$ & $0.4-35.5$ & 1.16 & & 20 & 298 & 2.57 & & & \\
\hline \multirow{4}{*}{ DMC } & hexano & 30 & $339-360$ & 101.3 & 0.73 & 0.004 & 36 & $298-318$ & 1.98 & 73 & $293-318$ & 0.63 \\
\hline & heptano & 30 & $355-369$ & 101.3 & 0.42 & 0.005 & 38 & $298-318$ & 1.42 & 58 & $293-318$ & 0.79 \\
\hline & octano & 33 & $362-396$ & 101.3 & 0.54 & 0.005 & 36 & $298-318$ & 1.82 & 72 & $293-318$ & 1.27 \\
\hline & nonano & 37 & $363-421$ & 101.3 & 2.68 & 0.008 & 17 & 318 & 2.91 & 83 & $293-318$ & 1.47 \\
\hline etanol & \multirow{3}{*}{ TAME } & 54 & $323-333$ & $30-59$ & 0.82 & 0.007 & 29 & 298 & 3.60 & 11 & 298 & 0.95 \\
\hline propanol & & 54 & $323-333$ & $13-44$ & 2.60 & 0.01 & 17 & 298 & 4.56 & 21 & 298 & 0.61 \\
\hline butanol & & 54 & $323-333$ & $6-42.5$ & 2.52 & 0.01 & 10 & 298 & 2.99 & & & \\
\hline metanol & \multirow{5}{*}{ DMC } & 104 & $314-362$ & $40-101.3$ & 0.49 & 0.003 & 15 & 313 & 4.10 & 24 & 313 & 0.56 \\
\hline etanol & & 152 & $325-362$ & $40-101.3$ & 0.78 & 0.012 & 30 & $303-313$ & 4.80 & 18 & 313 & 0.33 \\
\hline propanol & & 121 & $335-369.7$ & $40-101.3$ & 0.58 & 0.005 & 30 & $303-313$ & 3.23 & 18 & 313 & 0.15 \\
\hline butanol & & 55 & $337-388$ & $40-93.32$ & 0.86 & & 48 & $288-313$ & 3.06 & 45 & $288-313$ & 0.28 \\
\hline pentanol & & 26 & $364-410$ & 101.3 & 1.15 & 0.014 & 15 & 303 & 3.29 & 45 & $288-313$ & 0.4 \\
\hline \multicolumn{2}{|c|}{ Global } & 1370 & & & 0.99 & 0.0070 & 856 & & 3.85 & 754 & & 0.92 \\
\hline
\end{tabular}

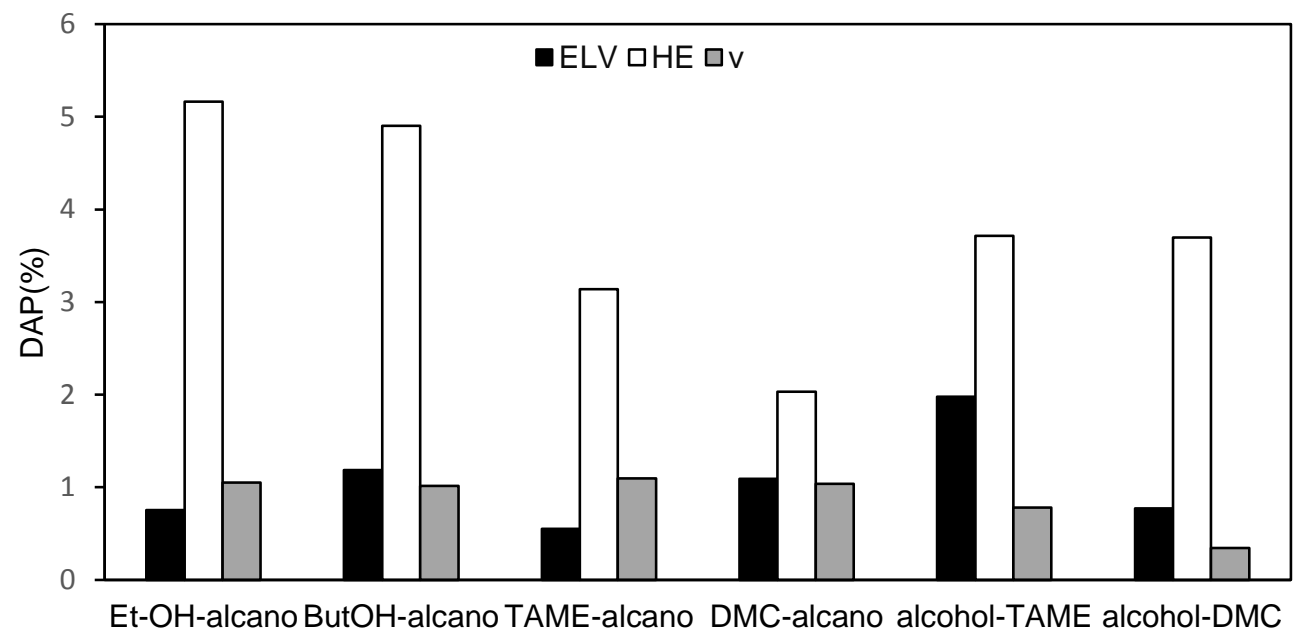

Fig. 1. Desviaciones medias a partir del modelo seleccionado para el ELV, la entalpía de exceso y el volumen de mezclas del tipo aditivo oxigenado-alcano y alcohol-aditivo oxigenado.

Para ilustrar el desempeño de las expresiones desarrolladas, en las Figuras 2 a 4 se presentan los resultados obtenidos para cada propiedad con algunas de las series trabajadas. 

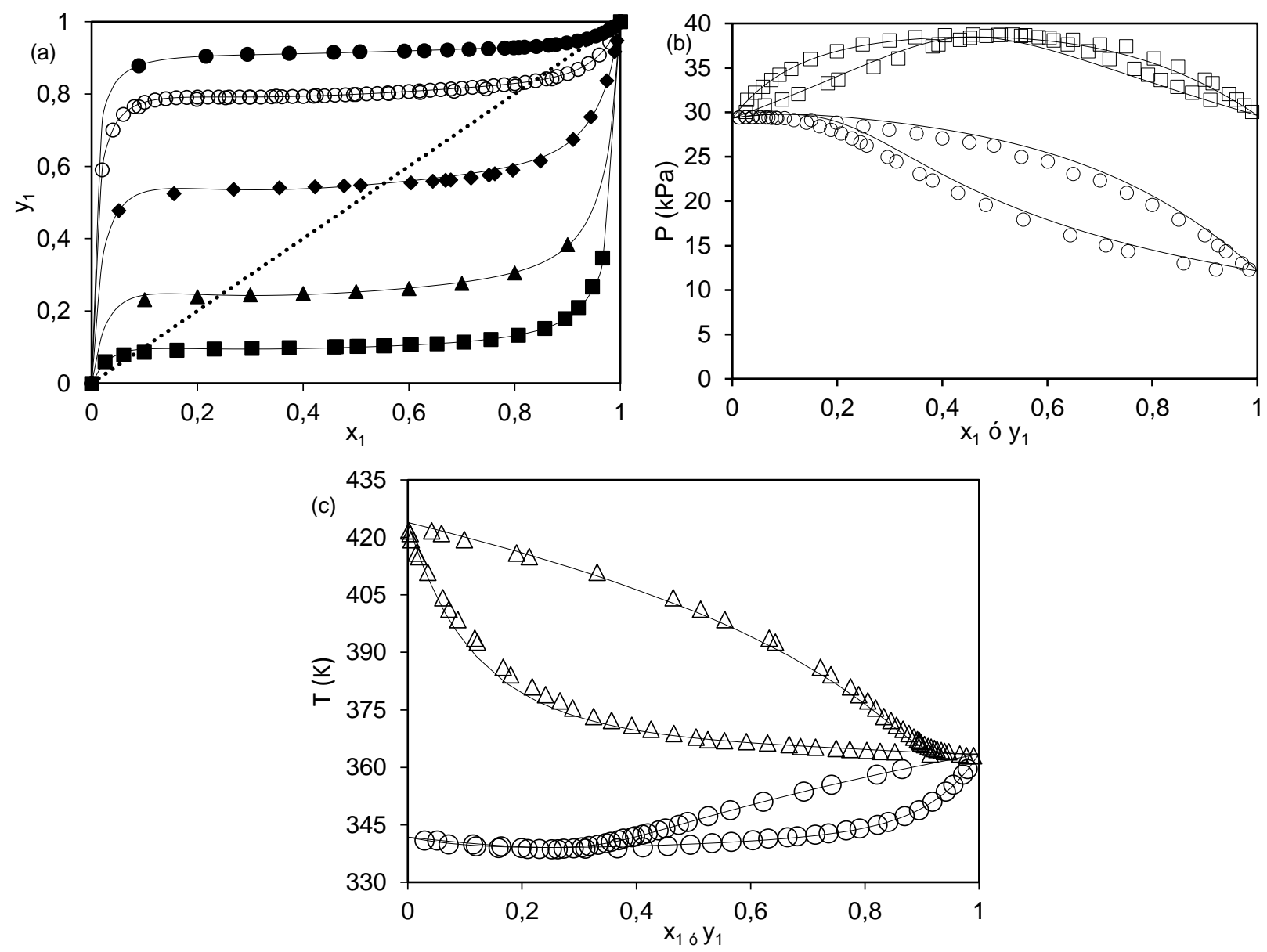

Fig. 2. Resultados de la nueva EdE para el ELV de las series: (a) - etanol- nC5, $\boldsymbol{\Delta}$ etanol- nC6, $\bullet$ etanol- $\mathrm{nC7}$, ○ etanolnC8, • etanol- nC9 (b) 口 TAME-nC $2 \mathrm{OH}$ a $323.15 \mathrm{~K}$, 。 TAME-nC ${ }_{3} \mathrm{OH}$ a $323.15 \mathrm{~K}$ (c) ○ DMC-nC, a $101.3 \mathrm{kPa}, \Delta \mathrm{DMC}-\mathrm{nC} 9$ a $101.3 \mathrm{kPa}$.

En la Figura 2 se reportan los resultados para el equilibrio líquido vapor. En el primer caso (Figura 2a) se presenta el diagrama $y_{1}$ vs $x_{1}$ para mezclas del tipo etanol (1) / $n$-alcano (2). Se ilustra que a través de expresiones generalizadas simples se puede describir cuantitativamente la variación en la composición azeotrópica en la medida que cambia el n-alcano. Se observa en la figura 2a que la predicción de los puntos azeotrópicos para mezclas etanol-alcano es correcta, aunque no se puede calcular la desviación porque el valor experimental específico del punto azeotrópico no se reporta. El segundo caso (Figura $2 b$ ) corresponde al diagrama Pxy a 323.15 K para la serie n-alcoholes-TAME. Para la mezcla etanol (1) / TAME (2) los valores calculados con la nueva EdE son prácticamente iguales a los reportados en la literatura (ver Tabla 3). Para la mezcla propanol (1) / TAME (2) se puede observar que existe diferencia entre los valores calculados y los experimentales. Sin embargo, la DAP en la presión de burbuja a $323.15 \mathrm{~K}$ es de $3.67 \%$.

El tercer caso (Figura 2c) corresponde al diagrama Txy a $101.3 \mathrm{kPa}$ para la serie $\mathrm{n}$-alcoholes-DMC. Para las 2 mezclas se puede observar que los valores calculados con el modelo propuesto son bastante cercanos a los reportados experimentalmente (ver Tabla 3). Los resultados son similares a los de la serie etanol-nalcanos, es decir, en la Figura $2 c$ se ilustra que es posible describir la presencia y variación del comportamiento azeotrópico dentro de una serie homóloga a partir de parámetros de las sustancias puras.

En la Figura 3 se ilustran los resultados para la entalpía de exceso de la serie etanol-n-alcanos (Figura 3a) y de la serie n-alcoholes-DMC (Figura 3b). Los casos reportados son aquellos que presentan la mayor y menor desviación de acuerdo con la Tabla 3. Los resultados de ambas series muestran la capacidad de la nueva EdE para describir la entalpía de exceso a partir de expresiones generalizadas simples. Para la serie etanoln-alcanos, se puede observar alguna diferencia entre los valores calculados y los datos experimentales en la zona diluida en etanol. De igual manera para la mezcla etanol (1) / nonano (2) se presenta una leve sobrepredicción con el modelo evaluado. Para la isoterma ilustrada en la Figura 3a la desviación media de la mezcla en cuestión es de $8.67 \%$. De acuerdo con Park et al. (2001) desviaciones hasta del $10.0 \%$ en la entalpía de exceso se pueden obtener cuando se modelan mezclas del tipo alcohol (1) / alcano (2) con una EdE del tipo asociación de fluido enmallado. Por otro lado, las entalpías de exceso calculadas son prácticamente iguales a aquellas reportadas en la literatura para la serie alcoholes-DMC. Los resultados de ambas series muestran que se puede calcular correctamente la entalpía de exceso de una mezcla binaria por medio de una EdE cúbica simple y a partir de parámetros que dependen solo de las sustancias puras. 

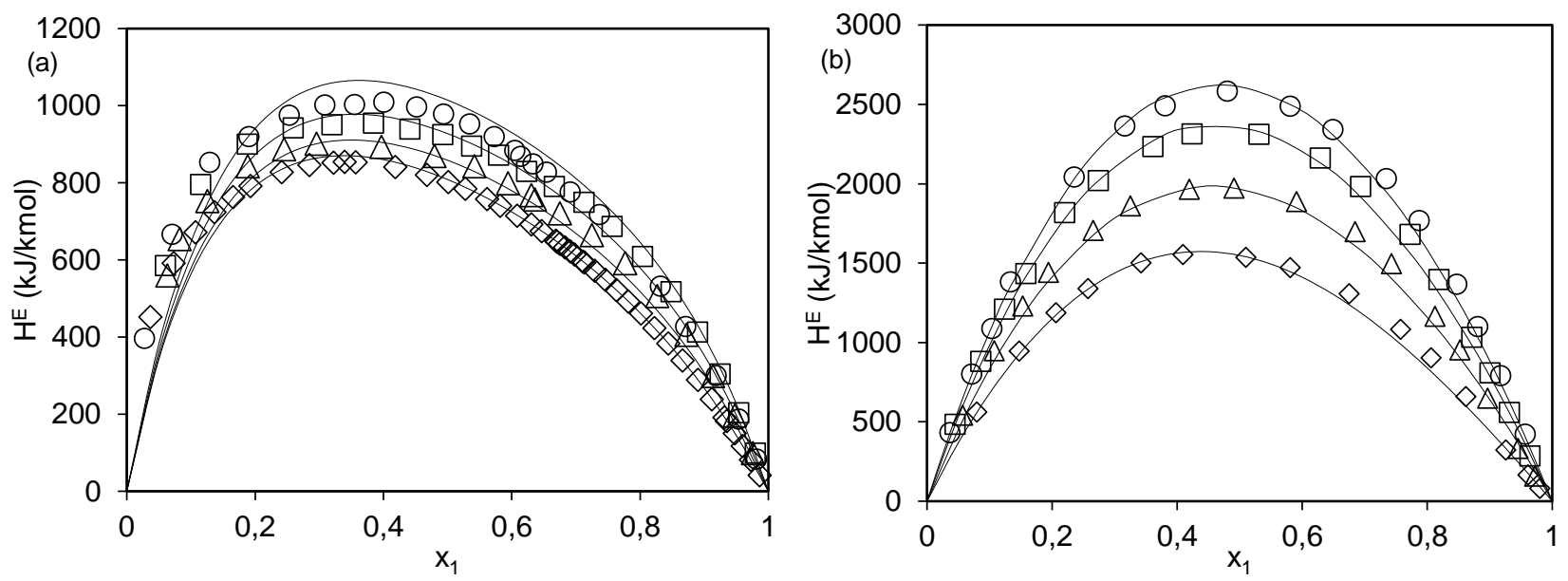

Fig. 3. Resultados para la entalpía de exceso de las series: (a) $\diamond$ etanol-nC 6 a $318.15 \mathrm{~K}, \Delta$ etanol-nC 7 a $318.15 \mathrm{~K}, \square$ etanol-nC 8 a $318.15 \mathrm{~K}$, 。 etanol-nC 9 a $318.15 \mathrm{~K}$. (b) $\diamond \mathrm{nC}_{1} \mathrm{OH}-\mathrm{DMC}$ a $313.15 \mathrm{~K}, \Delta \mathrm{nC}_{2} \mathrm{OH}-\mathrm{DMC}$ a $313.15 \mathrm{~K}, \square \mathrm{nC} \mathrm{OH}_{3}$ $\mathrm{DMC}$ a $313.15 \mathrm{~K}$, ○ $\mathrm{nC}_{4} \mathrm{OH}-\mathrm{DMC}$ a $313.15 \mathrm{~K}$.

Con respecto al volumen molar, en la Figura 4 se presenta la predicción de esta propiedad para mezclas formadas por DMC y las series de los n-alcanos y $n$-alcoholes respectivamente. Los resultados confirman la capacidad de la nueva EdE para describir el volumen molar de mezclas de diferente naturaleza. En los dos casos ilustrados se observa que los volúmenes calculados son muy cercanos a los reportados en la literatura. La desviación promedio de cada serie es menor al $1.0 \%$.
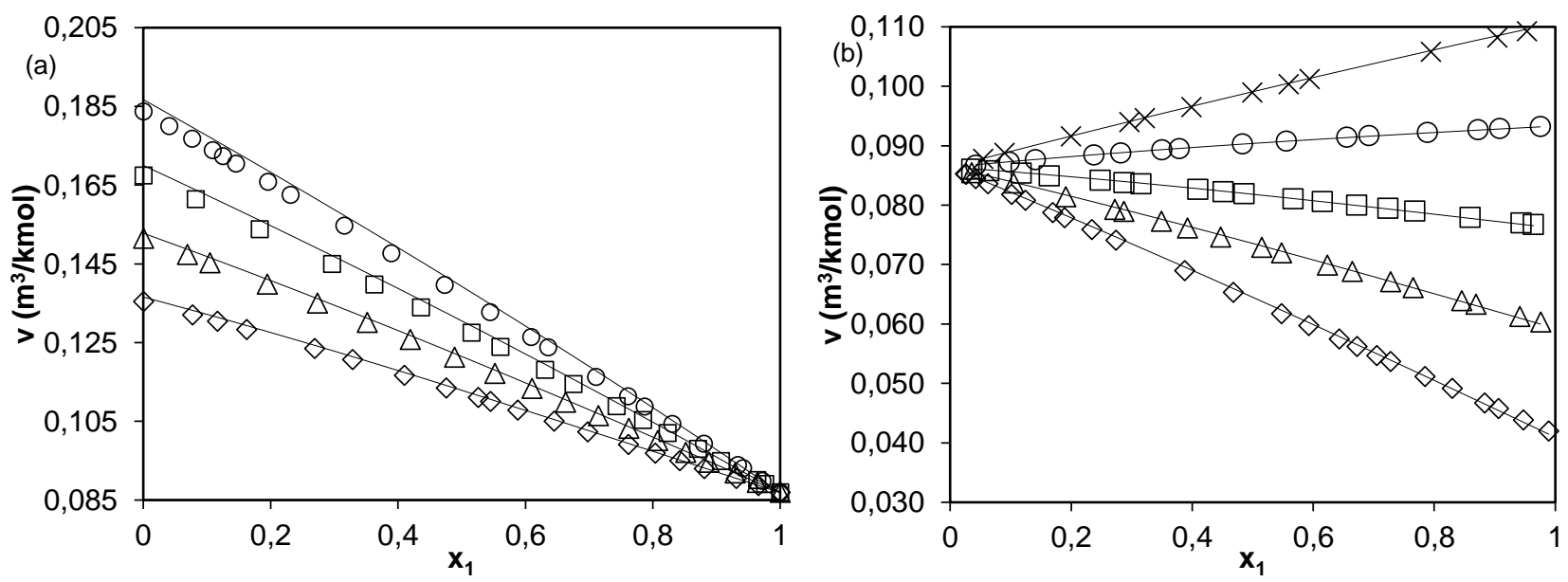

Fig. 4. Resultados para el volumen molar de las series: (a) $\diamond D M C-n C_{6}$ a $318.15 \mathrm{~K}, \Delta \mathrm{DMC}-\mathrm{nC}_{7}$ a $318.15 \mathrm{~K}, \square \mathrm{DMC}-\mathrm{nC} \mathrm{C}_{8} \mathrm{a}$ $318.15 \mathrm{~K}, \circ$ DMC-nC9 a $318.15 \mathrm{~K}$. (b) $\diamond \mathrm{nC}_{1} \mathrm{OH}-\mathrm{DMC}$ a $313.15 \mathrm{~K}, \Delta \mathrm{nC}_{2} \mathrm{OH}-\mathrm{DMC}$ a $313.15 \mathrm{~K}, \square \mathrm{nC} \mathrm{OH}_{3} \mathrm{OMC}$ a $313.15 \mathrm{~K}$, o $\mathrm{nC}_{4} \mathrm{OH}-\mathrm{DMC}$ a $313.15 \mathrm{~K}, \mathrm{x} \mathrm{nC}_{5} \mathrm{OH}-\mathrm{DMC}$ a $313.15 \mathrm{~K}$.

\section{COMPARACIÓN CON OTROS MODELOS DE LA LITERATURA}

Como se había mencionado antes, no existen muchos estudios acerca de la representación simultánea del equilibrio de fases y la entalpía de exceso. Sin embargo, para el caso de las mezclas alcohol-alcano hay al menos dos trabajos reportados en la literatura. En primer lugar, Shen y Lu (1997) reportaron resultados para 9 mezclas con la EdE de Peng-Robinson-Stryjek-Vera y las reglas de mezcla de Wong-Sandler. Por otra parte, Park et al. (2001) ajustaron la entalpía y el equilibrio de 8 mezclas alcohol-alcano con una ecuación de estado del tipo asociación de fluido enmallado. En la Tabla 4 se resumen las desviaciones reportadas en la literatura y los resultados obtenidos con la nueva EdE y las expresiones generalizadas de la Tabla 2.

En la primera parte se presentan los resultados para tres mezclas con el modelo evaluado y la EdE de asociación de Park et al. (2001). De acuerdo con los resultados, el equilibrio y la entalpía de exceso se describen correctamente con los dos modelos. Para la entalpía, los resultados parecen mejores con la nueva EdE. Las DAPs con el modelo de Park et al. (2001) se encuentran entre $3.61 \%$ y $8.88 \%$ mientras que las de la nueva EdE varían entre $5.20 \%$ y $6.15 \%$. Estos resultados muestran que para las mezclas analizadas, el desempeño de una EdE cúbica con reglas de mezclado del tipo $E d E+G E$ es comparable con el de un modelo termodinámico desarrollado para describir el fenómeno de asociación molecular. 
Tabla 4. Comparación de la nueva EdE con otras EdEs de la literatura.

\begin{tabular}{|c|c|c|c|c|c|c|}
\hline \multirow[b]{2}{*}{ Mezcla } & \multicolumn{3}{|c|}{ DAP P - ELV } & \multicolumn{3}{|c|}{ DAP $H^{E}$} \\
\hline & $\begin{array}{c}\text { Este } \\
\text { trabajo }\end{array}$ & $\begin{array}{c}\text { Park et al. } \\
(2001)\end{array}$ & $\mathrm{T}(\mathrm{K})$ & $\begin{array}{c}\text { Este } \\
\text { trabajo }\end{array}$ & $\begin{array}{c}\text { Park et al. } \\
(2001)\end{array}$ & $\mathrm{T}(\mathrm{K})$ \\
\hline \multirow{2}{*}{ etanol-pentano } & 0.7 & 1.54 & 303.15 & 6.15 & 3.61 & 298.2 \\
\hline & 3.25 & 2.01 & 422.6 & - & - & - \\
\hline \multirow{2}{*}{ etanol-hexano } & 0.4 & 0.47 & 298 & 5.92 & 8.75 & 283.2 \\
\hline & & & & 5.2 & 4.83 & 298.2 \\
\hline butanol-hexano & 1.57 & 2.5 & 298.15 & 5.88 & 8.88 & 298.2 \\
\hline \multirow[b]{2}{*}{ Mezcla } & \multicolumn{3}{|c|}{ DAP P - ELV } & \multicolumn{3}{|c|}{$\mathrm{DAP} \mathrm{H}$} \\
\hline & $\begin{array}{c}\text { Este } \\
\text { trabajo }\end{array}$ & $\begin{array}{c}\text { Shen y Lu } \\
\text { (1997) }\end{array}$ & $\mathrm{T}(\mathrm{K})$ & $\begin{array}{c}\text { Este } \\
\text { trabajo }\end{array}$ & $\begin{array}{c}\text { Shen y Lu } \\
\text { (1997) }\end{array}$ & $\mathrm{T}(\mathrm{K})$ \\
\hline etanol-hexano & 0.9 & 1.91 & 323 & 5.19 & 1.49 & 298 \\
\hline etanol-heptano & 0.8 & 0.54 & 303 & 2.08 & 5.55 & 298 \\
\hline butanol-pentano & 1.48 & 1.68 & 303 & 3.52 & 2.8 & 298 \\
\hline butanol-hexano & 2.01 & 2.31 & 298 & 5.99 & 1.85 & 298 \\
\hline butanol-heptano & 1.43 & 1.76 & 323 & 8.31 & 4.85 & 328 \\
\hline butanol-decano & 0.84 & 0.83 & 373 & 5.00 & 0.82 & 288 \\
\hline
\end{tabular}

En la segunda parte de la Tabla 4, se comparan dos ecuaciones de estado y dos reglas de mezclado del tipo $\mathrm{EdE}+\mathrm{G}^{\mathrm{E}}$ diferentes. En total se analizaron seis mezclas. Los resultados para el equilibrio son prácticamente iguales para las dos EdE. Las desviaciones reportadas por Shen y Lu (1997) para la entalpía de exceso son menores a las obtenidas con la nueva EdE. Solo para la mezcla etanol (1) / heptano (2) se obtienen mejores resultados con el modelo propuesto. La diferencia entre los dos modelos evaluados tiene sentido. Los resultados del trabajo de Shen y Lu (1997) corresponden al ajuste de una sola isoterma para el equilibrio líquido-vapor y otra para la entalpía de exceso de cada mezcla. Los resultados del modelo evaluado corresponden a la generalización de cada serie homóloga. Dicha generalización se realiza con el objetivo de describir varias isotermas para el equilibrio de fases y la entalpía de exceso.

Para demostrar que el desempeño de las dos ecuaciones es similar, la entalpía y el equilibrio de fases se correlacionaron para cada mezcla a las condiciones reportadas por Shen y Lu (1997). En la Figura 5, se presentan las desviaciones para la entalpía de exceso luego de ajustar los datos de las seis mezclas de la Tabla 4. Los resultados efectivamente muestran que la desviación del modelo evaluado disminuye cuando se analizan las mezclas individualmente. Adicionalmente, se puede observar que los dos modelos producen resultados similares. Para cuatro de las seis mezclas analizadas, la diferencia entre las DAPs de ambos modelos es menor al $1.00 \%$. Para la mezcla etanol (1) / heptano (2) el modelo evaluado es claramente superior que la EdE de Shen y Lu (1997). Finalmente, para la mezcla butanol (1) / decano (2) se obtienen mejores resultados con la EdE de Shen y Lu (1997).

En resumen, se puede establecer que el modelo propuesto es comparable con otros modelos disponibles en la literatura para representar el equilibrio de fases y la entalpía de exceso de mezclas complejas. A pesar de que los resultados pueden llegar a ser inferiores a modelos como el de Shen y Lu (1997), la principal ventaja de la nueva EdE consiste en que solo requiere el conocimiento de parámetros de las sustancias puras. Adicionalmente, si para algunas condiciones específicas se requieren mejores resultados, es posible ajustar el modelo a dichas condiciones. Finalmente, vale la pena aclarar que las expresiones generalizadas de la Tabla 2 se deben emplear preferiblemente a las condiciones utilizadas durante su desarrollo (ver Tabla 3). Orbey y Sandler (1996) demostraron que la capacidad de predicción de las reglas de mezclado del tipo $\mathrm{EdE}+\mathrm{G}^{\mathrm{E}}$ a altas temperaturas y presiones puede ser inadecuada cuando se ajustan simultáneamente datos de entalpía y equilibrio a bajas temperaturas. De acuerdo con Ohta (1996) y Ahlers y Gmehling (2002), es recomendable incluir todas las isotermas posibles durante el proceso de regresión de parámetros para obtener buenos resultados en amplios intervalos de temperatura. Debido a que no existen datos de equilibrio y/o entalpía de exceso a altas temperaturas para la mayoría de las mezclas que pertenecen a las series homólogas estudiadas, este enfoque no fue empleado para el modelo evaluado. 


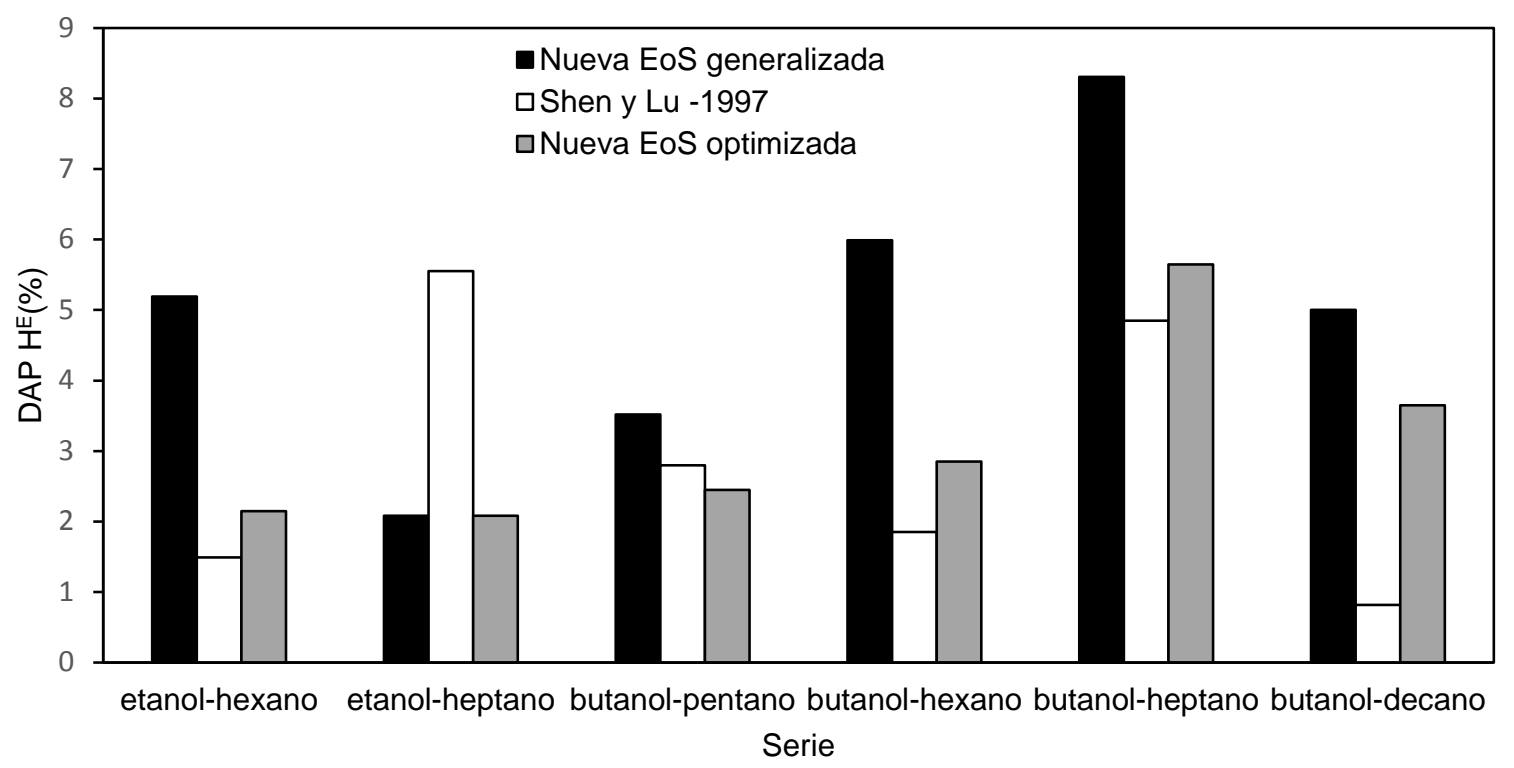

Fig. 5. Desviaciones para la entalpía de exceso de mezclas alcohol (1) / alcano (2) con la EdE VTPRH generalizada, la EdE VTPRH ajustada y la EdE de Shen y Lu (1997).

Por otro lado, existen resultados reportados en la literatura solo para el equilibrio líquido-vapor con diferentes modelos termodinámicos.

Por ejemplo, Forero y Velasquez (2014) utilizaron la EdE de Patel-Teja (Patel y Teja, 1981) con reglas de mezclado de Wong-Sandler (Wong y Sandler, 1992) y el modelo de actividad NRTL desarrollando expresiones generalizadas para los mismos tipos de mezclas del presente trabajo únicamente para el ELV. Se reportó una desviación global para la presión de burbuja de $1.09 \%$, siendo la DAP máxima de $2.43 \%$ para la mezcla propanol-TAME y una mínima de $0.21 \%$ para la mezcla TAME-hexano. Estos resultados son similares a los reportados en la tabla 3 para el modelo propuesto, cuya desviación global es $0.99 \%$ y las DAP máxima y mínima corresponden a $2.68 \%$ y $0.1 \%$ respectivamente. Es importante resaltar que los resultados del presente trabajo incluyen el ajuste adicional de la entalpía.

Para los casos de las series etanol-alcano y butanol-alcano, Bender et.al (2016) desarrollaron un método de contribución de grupos para el modelo PC-SAFT (Gross y Sadowski, 2001) aplicado al ELV. Reportaron una desviación global en la presión de burbuja de $5.02 \%$, siendo la DAP máxima de $9.11 \%$ para la mezcla butanolpentano y la mínima de $2.68 \%$ para etanol-hexano. Estas desviaciones son mayores a las del modelo propuesto, esto tiene sentido ya que es un método de contribución de grupos y no el ajuste de un pequeño grupo de mezclas (entre 3 y 5 por serie homóloga).

\section{CONCLUSIONES}

La ecuación de estado evaluada se puede utilizar para representar correctamente de manera simultánea propiedades como el equilibrio líquido-vapor, el volumen en fase líquida y la entalpía de exceso de mezclas complejas formadas por sustancias no polares y polares.

Los resultados son bastante buenos e ilustran la capacidad del modelo para representar las tres propiedades de forma simultánea para mezclas de aditivos oxigenados con alcanos y alcoholes. Las desviaciones globales son $0.99 \%, 3.85 \%$ y $0.92 \%$ para la presión de burbuja, la entalpía de exceso y el volumen molar respectivamente.

El modelo propuesto es comparable con otros modelos disponibles en la literatura para representar el equilibrio de fases y la entalpía de exceso de mezclas aditivos oxigenados-alcano y aditivos oxigenadosalcohol.

\section{NOTACIÓN}

\section{Símbolos}

a, b, c = parámetros de mezcla de la nueva ecuación de estado

$a_{i}, b_{i}, c_{i}=$ parámetros de la sustancia i de la nueva ecuación de estado

$\mathrm{R}=$ constante universal de los gases ideales $(\mathrm{kJ} / \mathrm{kmol} \mathrm{K})$

$\mathrm{T}=$ temperatura $(\mathrm{K})$ 
$\mathrm{P}=$ presión $(\mathrm{kPa})$

$\mathrm{v}=$ volumen molar $\left(\mathrm{m}^{3} / \mathrm{kmol}\right)$

$x_{i}=$ fracción molar de la sustancia $i$

$\mathrm{G}^{\mathrm{E}}=$ energía libre de Gibss de exceso $(\mathrm{kJ} / \mathrm{kmol})$

$A_{\mathrm{ji}}=$ parámetro NRTL relacionado con las energías de interacción entre las moléculas j e i $(\mathrm{kJ} / \mathrm{kmol})$

$\mathrm{H}_{1 \mathrm{i}}=$ parámetro 1 de la sustancia i para la función alfa de Heyen

$\mathrm{H}_{2 i}=$ parámetro 2 de la sustancia i para la función alfa de Heyen

$\beta_{\mathrm{ci}}=$ parámetro relacionado con la primera derivada de la función alfa a la temperatura crítica

$\gamma_{\mathrm{ci}}=$ parámetro relacionado con la segunda derivada de la función alfa a la temperatura crítica

$a_{\mathrm{ji}}, b_{\mathrm{ji}}, c_{\mathrm{ji}}=$ coeficientes para expresar $\mathrm{A}_{\mathrm{ji}}$ en función de la temperatura

$\mathrm{H}^{\mathrm{E}}=$ Entalpia molar de exceso $(\mathrm{kJ} / \mathrm{kmol})$

\section{Letras Griegas}

$\alpha_{i j}=$ parámetro NRTL de mezclado no aleatorio entre las sustancias i y j

$\omega_{i}=$ factor acéntrico de la sustancia $i$

$\chi_{i}=$ factor polar de la sustancia $i$

\section{Abreviaciones}

$\mathrm{EdE}=$ ecuación de estado

S.H. = serie homóloga

F.O. = función objetivo

$\mathrm{DMC}=$ dimetil carbonato

TAME = 2-metoxi-2-metilbutano

$\mathrm{NS}=$ número de sustancias

$\mathrm{ND}=$ número de datos

DAP = desviación absoluta promedio

$\mathrm{Y}=$ propiedad para la que se desea calcular el DAP (presión de burbuja, entalpía de exceso o volumen molar)

$\mathrm{D}=$ desviación

$E L V=$ equilibrio líquido-vapor

$\mathrm{HV}=$ Huron-Vidal

$\mathrm{NRTL}=$ Non random two liquid

\section{Súper/sub índices}

$c=$ condiciones críticas

exp $=$ experimental

sat $=$ condiciones de saturación

$b=$ condición de burbuja

$\mathrm{EdE}=$ calculado con la nueva EdE

$r=$ condiciones reducidas

$\mathrm{L}=$ propiedad en estado líquido

\section{REFERENCIAS}

Ahlers, J. y J. Gmehling, Development of a Universal Group Contribution Equation of State III. Prediction of Vapor-Liquid Equilibria, Excess Enthalpies, and Activity Coefficients at Infinite Dilution with the VTPR Model, Industrial \& Engineering Chemistry Research, 41 (23), 5890-5899 (2002)

Beatrice, C., G. Di Blasio y otros 10 autores, Technologies for energetic exploitation of biodiesel chain derived glycerol: Oxy-fuels production by catalytic conversion, Applied Energy, 102, 63-71 (2013)

Bender, N., N. Medeiros y R. de P. Soares. Avoiding binary interaction parameters in the GC-PC-SAFT model with a parametrization based in VLE and IDAC data: n-Alkanes and 1-alkanols, Fluid Phase Equilibria, 412, 9-20 (2016)

British Petroleum Company. BP Statistical Review of World Energy. 67ª Ed. Londres, Inglaterra (2018)

Cismondi, M. y J. Mollerup, Development and application of a three-parameter RK-PR equation of state, Fluid Phase Equilibria, 232, 74-89 (2005)

Collinet, E. y J. Gmehling, Activity coefficient at infinite dilution, azeotropic data, excess enthalpies and solid-liquid- 
equilibria for binary systems of alkanes and aromatics with esters, Fluid Phase Equilibria, 230, 131-142, (2005)

Djordjević, B., M. Kijevčanin y S. Šerbanović, Simultaneous presentation of VLE, HE and cpE by the PRSV equation of state with the modified van der Waals one-fluid and Huron-Vidal-Orbey-Sandler mixing rules, Fluid Phase Equilibria, 155, 205-218 (1999)

Escobedo-Alvarado, G. N. y S.I. Sandler, Prediction of Excess Enthalpies Using a Gex/EOS Model, Industrial \& Engineering Chemistry Research, 40 (4), 1261-1270 (2001)

Fantozzi, F., A. Frassoldati y otros 5 autores, An experimental and kinetic modeling study of glycerol pyrolysis, Applied Energy, 184, 68-76 (2016)

Forero G., L.A. y J.A. Velásquez J., A modified Patel-Teja cubic equation of state. Part II: Parameters for polar substances and its mixtures, Fluid Phase Equilibria, 364, 75-87 (2014)

Forero G., L.A. y J.A. Velásquez J., A generalized cubic equation of state for non-polar and polar substances, Fluid Phase Equilibria, 418, 74-87 (2016a)

Forero G., L.A. y J.A. Velásquez J., A simple and generalized model to represent the vapor-liquid equilibria and the liquidmolar-volume of alcohol-alkane binary mixtures, Fluid Phase Equilibria, 430, 112-134 (2016b)

Gross, J. y G. Sadowski, Perturbed-chain SAFT: an equation of state based on a perturbation theory for chain molecules, Industrial \& Engineering Chemistry Research 40,1244-1260, (2001)

Halm, R.L. y L.I. Stiel, A fourth parameter for the vapor pressure and entropy of vaporization of polar fluids, AIChE Journal, 13 (2), 351-355 (1967)

Heyen, G., A cubic equation of state with extended range of application, Proceedings of the 2nd World Congress of Chemical Engineering, Frankfurt, p. 5. (1980)

Huron, M.J. y J. Vidal, New mixing rules in simple equations of state for representing vapour-liquid equilibria of strongly non-ideal mixtures, Fluid Phase Equilibria, 3 (4), 255-271 (1979)

Kontogeorgis, G.M. y P. Coutsikos, Thirty Years with EoS/GE Models—What Have We Learned?, Industrial \& Engineering Chemistry Research 51 (11), 4119-4142 (2012)

Ohta, T., Representation of excess enthalpies by the PRSV equation of state with the modified Huron-Vidal first order and Wong-Sandler mixing rules, Fluid Phase Equilibria,129, 89-103 (1997)

Orbey, H. y S.I. Sandler, A comparison of various cubic equation of state mixing rules for the simultaneous description of excess enthalpies and vapor-liquid equilibria, Fluid Phase Equilibria, 121, 67-83 (1996)

Patel, N. C., A. S. Teja, A new cubic equation of state for fluids and fluid mixtures, Chemical Engineering Science 37(3), 463-473 (1982)

Park, B.H., J.W. Kang y otros dos autores, An explicit hydrogen-bonding non-random lattice-fluid equation of state and its applications, Fluid Phase Equilibria 183-184, 111-119 (2001)

Renon, H. y J.M. Prausnitz, Local compositions in thermodynamic excess functions for liquid mixtures, AIChE J., 14 (135), 135-144 (1968)

Šerbanović, S., I. Grgurić y otros 3 autores, Thermodynamic modeling of vapor-liquid equilibria and excess properties of the binary systems containing diethers and n-alkanes by cubic equation of state, Korean Journal of Chemical Engineering, 21, 858-866 (2004)

Shen, S. y B. Lu, On the simultaneous representation of vapor-liquid equilibria and excess enthalpies of 1-alkanol $+n$ alkane mixtures, Chemical Engineering Communications, 159 (1), 1-15 (1997)

Valderrama, J. The State of the Cubic Equations of State. Industrial \& Engineering Chemistry Research, 42 (8), 1603-1618 (2003)

Velásquez J., J.A. y L.A. Forero G., Calculation of complex phase equilibria of DMF/alkane and acetonitrile/alkane systems using a modified Peng-Robinson EoS and the Huron-Vidal mixing rules, Journal of Molecular Liquids, 243, 600-610 (2017)

Voutsas, E., V. Louli y otros 3 autores. Thermodynamic property calculations with the universal mixing rule for EoS/GE models: Results with the Peng-Robinson EoS and a UNIFAC model, Fluid Phase Equilibria, 241, $216-228$ (2006)

Wong, D.S.H. y S.I. Sandler, A theoretically correct mixing rule for cubic equations of state, AIChE 38( 5), 671-680, (1992)

Zhao,Y., X. Dong y otros 4 autores. Evaluation of PR, NRTL, UNIFAC, and PCSAFT on the VLE of Binary Systems Containing Ammonia, Industrial \& Engineering Chemistry Research, 56 (8), 2287-2297 (2017) 
